\title{
EXPERIMENTAL SETTING FOR TESTING THE BOND STRENGTH BETWEEN METAL ALLOY AND PEEK
}

\author{
Zhivko Georgiev, Svetlin Alexandrov, Zlatina Tomova, \\ Department of Prosthetic Dentistry, Faculty of Dental Medicine, Medical Uni- \\ versity Plovdiv, Bulgaria.
}

\begin{abstract}
Purpose: The aim of the article is to present an experimental setting for bending strength testing of metal specimens covered with PEEK.

Materials and methods: Three groups of metal specimens made of cobalt chromium alloy are produced using the lost wax technique: group 1 - specimens with a smooth metal surface; group 2 - sand blasted metal surface; group 3 - sand blasted metal surface with retention crystals. The shape and size of the specimens are designed to correspond to the retentive elements of the apparatus for micro tension and micro pressure LMT 100. PEEK is pressed over the specimens, and after thermal cycling, the bond between metal alloy and PEEK is studied using bending strength three-point micro testing.

Results and discussion: The experimental setting is focused on the type of specific contact surface between the two materials. The design corresponds to the requirements of apparatus LMT 100 and allows the production of a big number of specimens. Because of the thermal regime of BioHPP pressing, it is impossible to add groups of specimens using contemporary adhesives on the metal surface.

Conclusion: Created specimens allow strength investigation by three-point bending micro testing. The experimental setting provides the production of a big number of specimens with the same shape and size.
\end{abstract}

Keywords: specimens, PEEK, experimental setting

\section{INTRODUCTION}

BioHPP is a thermoplastic high technology polymer on the base of PEEK. The mechanical and biological properties of the material make it suitable for medical and dental use. In the field of dental medicine, it can be used for removable and fixed prosthetic restorations, the production of individual implants and implant abutments, orthodontic wires, and obturators $[1,2,3]$. The strength of the connection between PEEK and other dental materials is important for the durability and period of use of the dental constructions $[4,5]$. Specific treatment of the contact surfaces improves the bond strength between PEEK and other materials $[6,7,8]$. For strength studies of the dental materials, different methods and experimental settings are used $[9,10,11,12]$. At bending strength measurements, one part of the specimen is exposed to pressure, and the other is exposed to tension (stretching).

\section{Aim}

Developing our own experimental setting for bending strength testing of metal specimens covered with PEEK.

\section{MATERIALS AND METHODS}

- Profile bar wax - a mixture of natural waxes with hard consistency needed for facilitated work. Measurements: $2.00 \mathrm{~mm}$ X $1.00 \mathrm{~mm}$, Dentaurum, Germany.

Fig. 1. Cristalloy M (Sandental Ltd., Bulgaria) - cobalt chromium alloy.

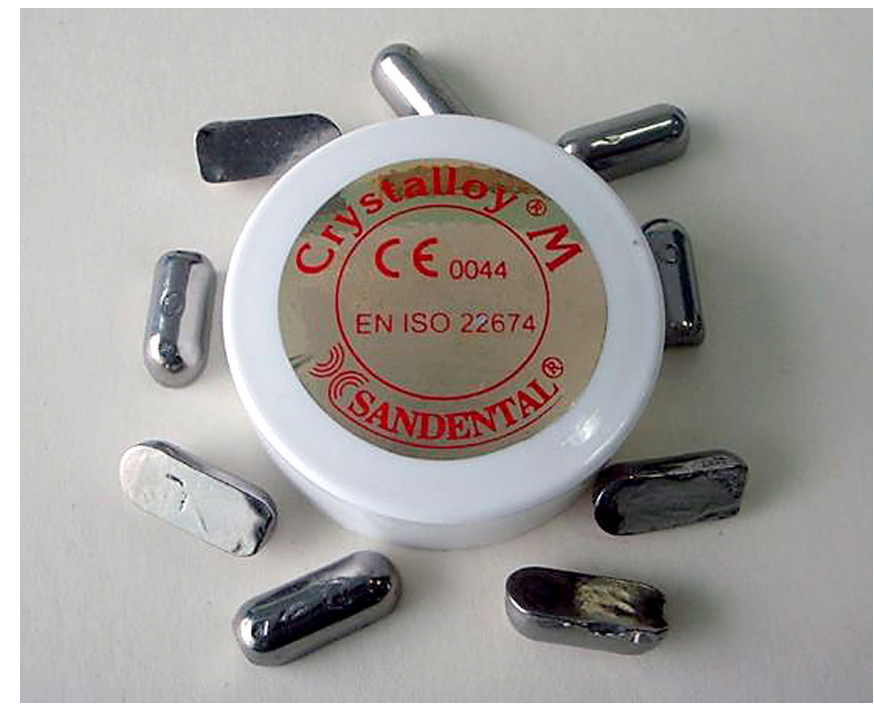

- Cobalt chromium base alloy - Cristalloy M (Sandental) (Fig.1). The content of the alloy is: Co - 66,5\%, $\mathrm{Cr}-27 \%, \mathrm{Mo}-5 \%, \mathrm{Si}-0,5 \%, \mathrm{Mn}-1 \%$. Density - 8,1 g/ $\mathrm{mm} 3$, Hardness $\mathrm{H}_{\mathrm{B}}-215$, melting interval $-1270-1210^{\circ} \mathrm{C}$, casting temperature $-1365^{\circ} \mathrm{C}$, tensile strength $-570 \mathrm{~N} / \mathrm{mm}^{2}$

- PEEK, BioHPP (Bredent, Germany) - specially optimized biopolymer for dental use (Fig.2). Due to the reinforcement with specific ceramic filling (20\% of the full volume, size of particles $0.3-0.5 \mu \mathrm{m}$ ), it has optimized mechanical properties and high polishing abilities. 
Fig. 2. BioHPP granules.

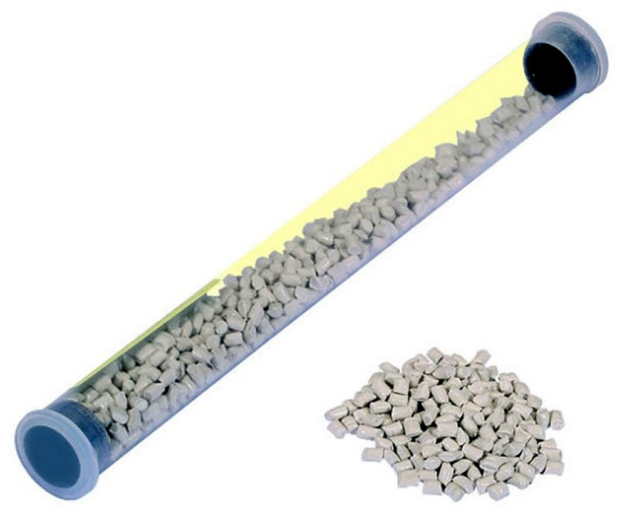

Specimens are produced by the classical method of the lost wax technique (Fig. 3, 4) with the following sequence: Profile bar wax is used to produce the metal bars. The length of each specimen is $25 \mathrm{~mm}$.

Fig. 3. Standard crucible with wax prototypes.

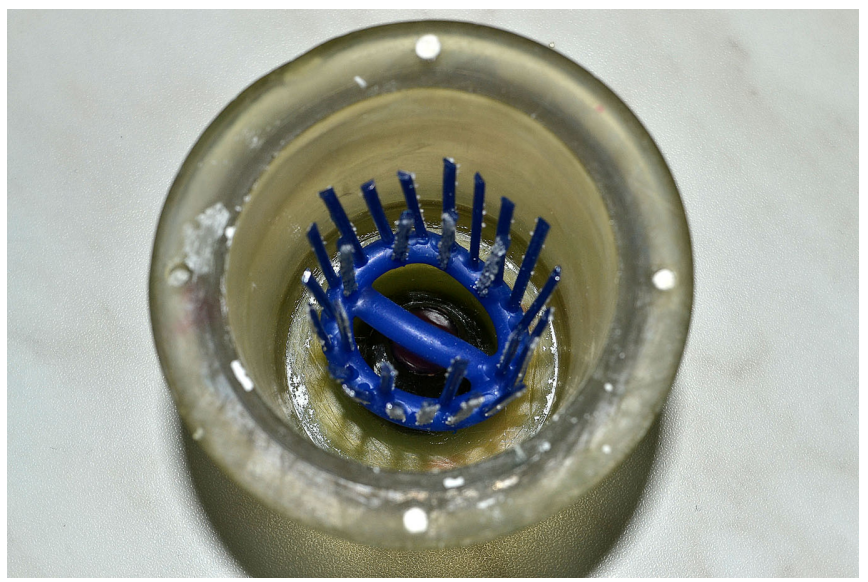

Fig. 4. Casted metal bars.

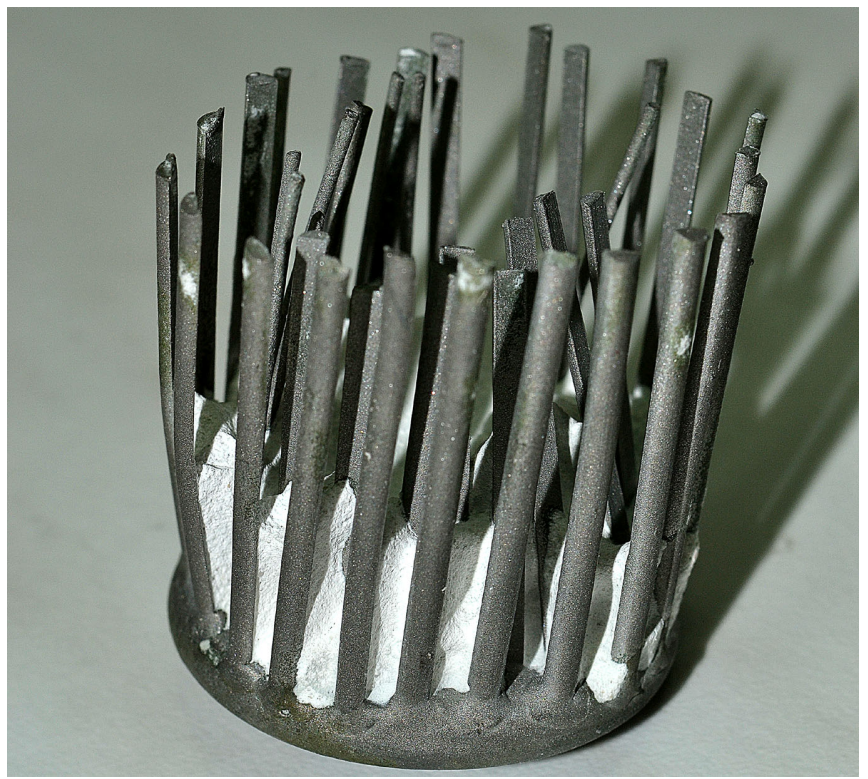

The specimens are divided into three groups according to the configuration of the metal surface:

Group 1 - Smooth metal surface

Group 2 - Sand blasted metal surface

Group 3 - Sand blasted metal surface with retentive crystals - Retention crystals $0,5 \mathrm{~mm}$, Bredent, Germany.

Preparation for pressing PEEK: A wax area with size $7 \mathrm{~mm}$ X $2 \mathrm{~mm}$ is modeled over the casted metal bars and replaced with the biopolymer by pressing (Fig. 5).

Fig. 5. Specimens prepared for investing.

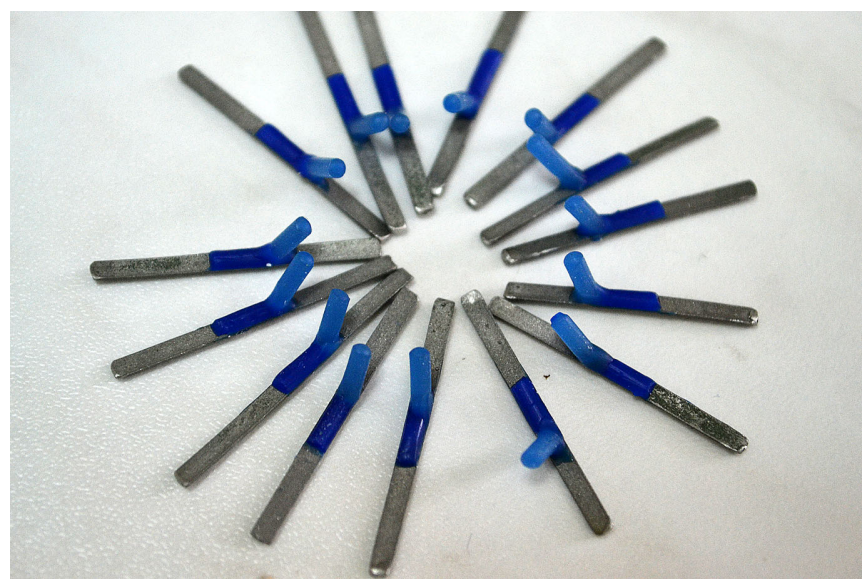

The wax modeled metal specimens are fixed to a bar of a profile wax, and the whole composition is carefully placed into the casting crucible (Fig. 6).

Fig. 6. Specimens fixed into the crucible.

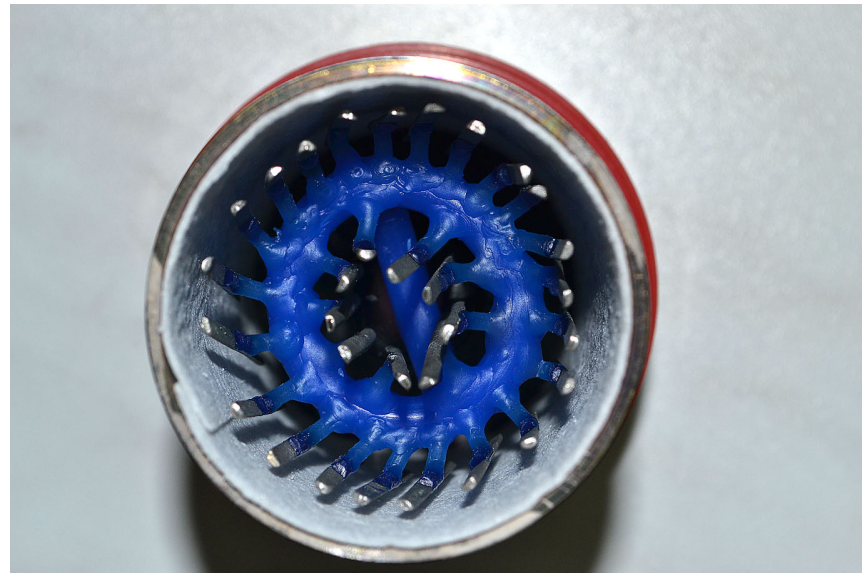

After production of the specimens, thermocycling is done using thermocycler LTC 100, LAM Technologies, Italy.

For the bending strength tests, the apparatus LMT100 (LAM Technologies, Italy), present at the Department of Prosthetic dentistry, FDM, MU - Plovdiv, is used. The designed retentive elements for the apparatus with fixing and a loading part are made of multicomponent steel (Fig.7). 
Fig. 7. Retentive elements for LMT 100

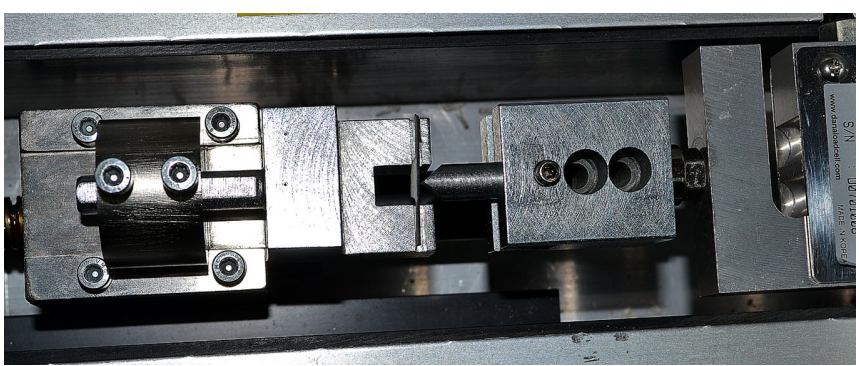

Fig. 8 Testing of the bond strength

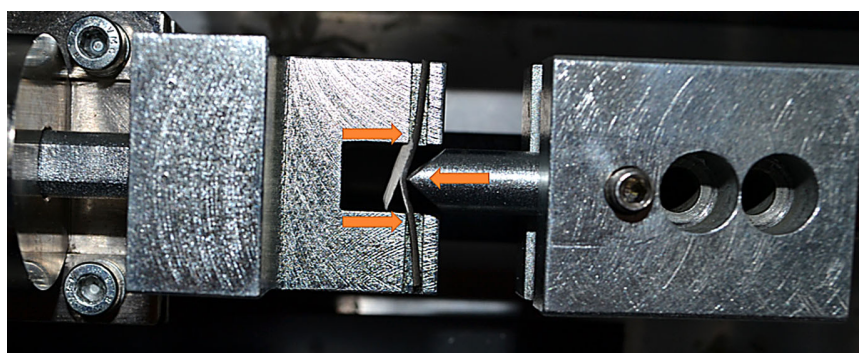

\section{RESULTS}

ISO standards for processing and use of dental materials are followed while making the specimens. After the casting of the metal alloy, the biopolymer is pressed over the metal surface. The specimens received after the strict completion of the laboratory protocol for pressing BioHPP over a base metal alloy are shown in Fig. 8. Three groups of 35 specimens each are produced. The size of each specimen is $2.00 \mathrm{~mm} \mathrm{X} 1.00 \mathrm{~mm} \mathrm{X} 25.00 \mathrm{~mm}$.

Fig. 9. BioHPP pressed over metal bars.

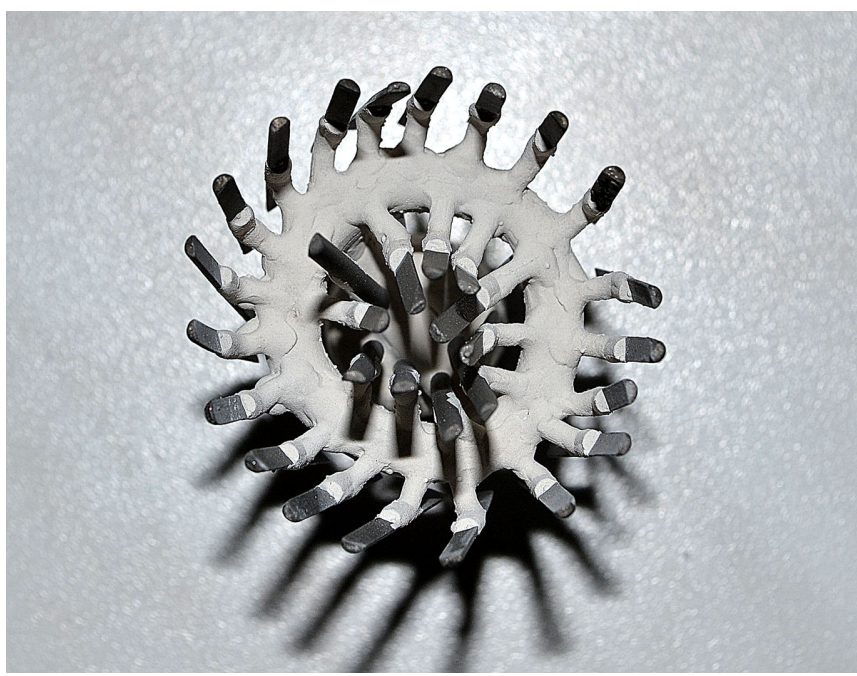

\section{DISCUSSION}

An important point at the creation of the experimental setting is that the size of the specimens must correspond to the size of the fixing retentive element of the apparatus LMT 100 for micro tension and micro pressure (LAM Technologies, Italy). The created experimental setting provides quick fixing of specimens in the desired position. The setting is focused on the type of the specific contact surface between the materials because the main purpose of the study is to measure the bond strength using the three-point micro test. The method of testing and the apparatus present at the department of prosthetic dentistry of MU Plovdiv define the size and the shape of the specimens. The retentive micro shapes in two of the study groups are classic and have proven their reliability in practice. It is impossible to add a group of test specimens using contemporary adhesive systems like polymers and metal primers because it is possible to harm or to burn the surface adhesive during the thermal regime of BioHPP pressing.

\section{CONCLUSION}

The created specimens allow strength measurements by three-point bending micro testing. The experimental setting provides the production of a big number of specimens with the same shape and size. We recommend the method for other static strength tests and for exploring the bond strength between two different types of materials. Further investigations of the reliability of the bond between BioHPP and the surface of the base metal alloy may include groups with retentive elements (crystals burnt without ash residue) with irregular shape on the metal surface, sand

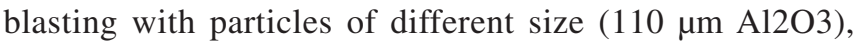
laser and acid preparation of the metal surface. 


\section{REFERENCES:}

1. Abu Bakar MS, Cheng MH, Tang SM, Yu SC, Liao K, Tan CT, et al. Tensile properties, tension-tension fatigue and biological response of polyetheretherketone-hydroxy apatite composites for load-bearing orthopedic implants. Biomaterials. 2003 Jun;24(13): 2245-50. [PubMed]

2. Georgiev J, Vlahova A, Kissov H, Aleksandrov S, Kazakova R. Possible application of BioHPP in prosthetic dentistry: a literature review. $J$ of IMAB. 2018 Jan-Mar;24(1):1896-1898. [Crossref]

3. Georgiev Zh. [Combined Prosthetic restorations made of PEEK and metal alloys]. [Dissertation] Medical University Plovdiv. 2018. 47-79. [in Bulgarian].

4. Bechir ES, Bechir A, Giora C, Manu R, Burcea A, Dascalu IT. The Advantages of BioHPP Polymer as Superstructure Material in Oral Implantology. Mater Plast. 2016 Sep;53(3): 394-398. [Internet]

5. Schwitalla AD, Spintig T, Kallage I, Müller WD. Pressure behavior of dif- ferent PEEK materials for dental implants. J Mech Behav Biomed Mater. 2016 Feb;54:295-304. [PubMed]

6. Schwitalla AD, Spintig T, Kallage I, Müller WD. Flexural behavior of PEEK materials for dental application. Dent Mater. 2015 Nov;31(11): 1377-84. [PubMed]

7. Fuhrmann G, Steiner M, FreitagWolf S, Kern M. Resin bonding to three types of polyaryletherketones (PAEKs)-durability and influence of surface conditioning. Dent Mater. 2014 Mar;30(3):357-63. [PubMed]

8. Hallmann L, Mehl A, Sereno N, Hämmerle CHF. The improvement of adhesive properties of PEEK through different pre-treatments. Applied Surface Science. 2012 Jul 1;258(18): 7213-18. [Crossref]

9. Kern M, Lehmann F. Influence of surface conditioning on bonding to polyetheretherketon (PEEK). Dental Mater. 2012 Dec;28(12):1280-3. [PubMed]

10. Boinard E, Pethrick R, MacFarlane $\mathrm{C}$. The influence of thermal his- tory on the dynamic mechanical and dielectric studies of polyetheretherketone exposed to water and brine. Polymer. 2000 Feb;41(3):1063-76. [Crossref]

11. Sarot JR, Contar CM, Cruz AC, de Souza Magini R. Evaluation of the stress distribution in CFR-PEEK dental implants by the three-dimensional finite element method. J Mater Sci Mater Med. 2010 Jul;21(7):2079-85. [PubMed]

12. Sproesser O, Schmidlin PR, Uhrenbacher J, Roos M, Gernet W, Stawarczyk B. Effect of sulfuric acid etching of polyetheretherketone on the shear bond strength to resin cements. J Adhes Dent. 2014 Oct;16(5):465-72. [PubMed]

13. Wimmer T, Huffmann AM, Eichberger M, Schmidlin PR, Stawarczyk B. Two-body wear rate of PEEK, CAD/ CAM resin composite and PMMA: Effect of specimen geometries, antagonist materials and test set-up configuration. Dent Mater. 2016 Jun;32(6): e127-36. [PubMed]

Please cite this article as: Georgiev Z, Alexandrov S, Tomova Z. Experimental setting for testing the bond strength between metal alloy and PEEK. J of IMAB. 2021 Jul-Sep;27(3):3881-3884.

DOI: https://doi.org/10.5272/jimab.2021273.3881

Received: 12/01/2021; Published online: 17/08/2021

\author{
Address for correspondence: \\ Zhivko Georgiev \\ Department of Prosthetic Dentistry, Faculty of Dental Medicine, Medical Uni- \\ versity Plovdiv, \\ 15 A, Vasil Aprilov blv., 4000 Plovdiv, Bulgaria \\ E-mail: Jivko_169@abv.bg
}

